\title{
Aportes de los doctorados de educación en ciencia, tecnología y sociedad, desde la sistematización de sus investigaciones doctorales científicas y formativas, $2000-2010^{1}$
}

\author{
claudia Vélez de la Calle Ph.D. ${ }^{2}$, Marta Osorio Malaver Ph.D. ${ }^{3}$ \\ José Duván Marín Gallego Ph.D4., Mg. Mercedes Rodríguez ${ }^{5}$ \\ Juan Carlos Flórez, Carmen Edith Henao \\ Adelina Peña y Reina Saldaña
}

Fecha de recepción: 21 de julio de 2013

Fecha de revisión: 9 de agosto de 2013

Fecha de aprobación: 10 de septiembre de 2013

1 Artículo final fruto de investigación interinstitucional financiada entre el Doctorado en Educación de la Universidad Santo Tomás (Bogotá) y la Universidad San Buenaventura (Cali), en los Programas de Maestría y Doctorado en Educación. La investigación está en consonancia con las investigaciones sobre ciencia, tecnología y sociedad y sus aportes a la consolidación de las teorías pedagógicas con impacto en el desarrollo social del país.

2 Doctora en Filosofía y Ciencias de la Educación, docente directiva Universidad San Buenaventura (Cali), autora de numerosos artículos y varios libros sobre el tema de educación, epistemología y filosofía. Correo electrónico claudiavelez@ustadistancia.edu.co

3 Doctora en Pedagogía de la Universidad de Granada-España. Realizó estudios posdoctorales en narración de textos científicos. Se encuentra inscrita en la Línea de Organización y Gestión Educativa en Colciencias. Se desempeña como decana de la Facultad de Educación y directora del Doctorado en Educación de la Universidad Santo Tomás en Bogotá- Colombia. Correo electrónico. martaosorio@ ustadistancia.edu.co

4 Doctor en Educación, Universidad de Newport, Florida, docente-investigador de la Universidad Santo Tomás de Bogotá, autor de numerosos artículos sobre el tema de educación y epistemología, director del Grupo Currículo y Evaluación. Correo: joseduvanmarin@ustadistancia.edu.co

5 Magister en pedagogía, candidata a doctor en instituciones, profesorado y currículo, inscrita a la línea de Organización y Gestión Educativa. Docente de la Maestría en Educación de la Universidad Santo Tomás en Bogotá- Colombia. Correo electrónico: mercedesrodriguez@ustadistancia.edu.co 


\section{Resumen}

Este artículo es resultado de la investigación interinstitucional entre el Doctorado en Educación de la Universidad Santo Tomás y la Universidad San Buenaventura de Cali, programa de Maestría en Educación: Desarrollo Humano y el proyecto de Doctorado en Educación. Se hace una síntesis de lo que se encontró a través de la búsqueda en los documentos de los doctorados en educación en Colombia, durante el periodo 2000-2010, con relación a las investigaciones sobre ciencia, tecnología y sociedad, y sus aportes a la consolidación de las teorías pedagógicas con impacto en el desarrollo social del país.

\section{Palabras Clave}

Investigación, ciencia, tecnología, sociedad, desarrollo, educación, pedagogía.

\section{Contributions of doctoral education in science, technology and society, from the systematization of their doctoral research scientific and formative, 2000-2010}

\section{Abstract}

This paper is the result of inter-institutional research doctorate in education from the University of St. Thomas and St. Bonaventure University in Cali, Master of Education program: Human Development and the proposed Doctorate in Education. It is a synthesis of what was found by searching the documents Doctorate in Education in Colombia during the period 2000-2010, in relation to research on science, technology and society and their contributions to the consolidation of pedagogical theories impact on the country's social development.

\section{Keywords}

Research, science, technology, society, development, education, pedagogy. 


\section{Contribuições dos doutorados em educação em ciência, tecnologia e sociedade, desde a sistematização das suas pesquisas de doutorado científicas e formativas 2000-2010}

\section{resumo}

Este artigo é o resultado de investigação interinstitucional do Doutorado em Educação da Universidade Santo Tomas e da Universidade San Buenaventura Cali, programa de Mestrado em Educação: Desenvolvimento Humano e o projeto de Doutorado em Educação. Faz-se uma síntese do que foi encontrado através da busca nos documentos dos doutorados em educação na Colômbia, durante o período 20002010, em relação com as pesquisas sobre a ciência, a tecnologia e a sociedade, e suas contribuições para a consolidação das teorias pedagógicas com impacto no desenvolvimento social do país.

\section{Palavras-chave:}

Pesquisa, ciência, tecnologia, sociedade, desenvolvimento, educação, pedagogia.

\section{Introducción}

La investigación Aportes de los doctorados de educación en ciencia, tecnología y sociedad, desde la sistematización de sus investigaciones doctorales científicas y formativas, 2000-2010, es un proyecto de cooperación interinstitucional realizado entre el programa de Doctorado en Educación de la Universidad Santo Tomás y la Maestría en Educación: Desarrollo Humano, y el proyecto de Doctorado en Educación que está construyendo la Universidad San Buenaventura de Cali, con el propósito de indagar sobre los aportes investigativos en ciencia, tecnología y sociedad de los doctorados en educación en Colombia en la década pasada, y su contribución en la consolidación de una teoría social pedagógica que impacte en el desarrollo social y económico del país. 
Los estudios de ciencia, tecnología y sociedad datan de los años 40 en adelante del siglo XX, en torno a las discusiones sobre el uso de la ciencia, a propósito de los enfrenamientos cruentos de la Segunda Guerra Mundial y, posteriormente, la Guerra Fría, entre capitalismo y comunismo (EE UU y Unión Soviética). El exterminio de vidas que ocasionó la explosión de la bomba atómica en Hiroshima y Nagasaki, generó reacciones y movimientos multitudinarios de protesta que impusieron con mayor fuerza el debate de la relación ciencia-tecnología y ética. La década que ya concluyó del siglo XXI, retomó con más fuerza este debate por la irrupción de la revolución de la informática, la mediática, la biotecnología, la biopolítica y la geopolítica actuales.

Los procesos de crecimiento de las ciudades, los desarrollos de la tecnología que aceleró sus ventas y usos, obligó, tanto a los países mal llamados del "primer" mundo, como del "tercer" mundo, a una adquisición rápida de competencias modernas en este campo, como en el de la ciencia y en el del uso de una segunda lengua, generando en la educación una demanda activa de nueva alfabetización masiva en ciencias y tecnología.

De esta manera, las universidades y los centros de educación media, vocacional y superior, tuvieron que adaptarse a estas nuevas competencias en sus finalidades educativas y curriculares y, para ello, asumieron los enfoques de didácticas de las ciencias en su componente de enseñabilidad y aprendizaje interactivo. Morell Alonso (2009), nos recuerda que "Las problemáticas de la enseñanza de las ciencias deben relacionarse con las problemáticas sociales de la educación. Este enfoque en CTS corresponde al campo de los estudios en didácticas de las ciencias y estudios sociales en ciencia y tecnología" (p.10).

La enseñanza de las ciencias no es un ejercicio didáctico independiente del contexto social, económico y político, pues, tal como argumenta Lawler (en Aibar y Quintanilla, 2012), la noción de desarrollo no debe ser interpretada "exclusivamente desde el ámbito de la economía para extenderse hacia otros asuntos como, por ejemplo, la cohesión social, la equidad y la protección del medio ambiente" (p. 164). Por lo tanto, una enseñanza de las ciencias, y particularmente en los doctorados, a la vez que piensa en las racionalidades investigativas, también tiene como reto proyectar los impactos que en la vida socio-ambiental de este planeta se generan.

Los anteriores motivos y argumentos, sumados a la pertinencia que se exige del conocimiento, fueron suficientes para que las instituciones citadas iniciaran un proceso investigativo que diera razones acerca de qué han hecho los doctorados en educación en la década recién terminada, en relación con uno de los problemas 
más importantes del mundo moderno: el desarrollo de la ciencia y la tecnología, y su impacto en el desarrollo social.

\section{El problema y sus objetivos}

El proyecto partió de la formulación del problema que quedó plasmado en la siguiente pregunta: ¿Cuáles son las tendencias investigativas de los doctorados en educación en Colombia desde 2000 a 2010, en el campo de la ciencia, la tecnología y sociedad, y qué aportes han hecho, mediante la construcción de sus teorías pedagógicas, a la calidad de la educación y al desarrollo social y económico del país?

El planteamiento de esta pregunta dio lugar a la formulación de los siguientes objetivos:

Objetivo general. Identificar las tendencias investigativas en ciencia, tecnología y sociedad de los doctorados en educación en Colombia desde el 2000 a 2010, y su contribución, mediante las construcciones teóricas sobre pedagogía, a la calidad de la educación y al desarrollo social y económico del país.

\section{Objetivos específicos}

1. Determinar las relaciones entre educación ciencia y tecnología.

2. Documentar la producción y la productividad investigativa en Colombia de los doctorados en educación en ciencia, tecnología y sociedad, desde los años 2000 a 2010.

3. Establecer las tendencias y los aportes investigativos en ciencia, tecnología y sociedad de los doctorados en educación a través de la construcción de sus teorías pedagógicas.

4. Relacionar críticamente la incidencia y el impacto de las teorías pedagógicas construidas por los doctorados en la calidad de la educación y en el desarrollo sostenible del país. 


\section{El proceso metodológico}

La investigación se inicia con el planteamiento del problema, toda vez que es una investigación cualitativa. No se trata solamente de describir los hechos, sino que era importante asumir una posición crítica frente a lo que estaba sucediendo: por eso, el enfoque epistémico es el crítico social, inspirado en los postulados de la Escuela de Frankfort sobre la Teoría crítica de la sociedad, que considera todo el conocimiento como mediado social e históricamente y, a partir de una revisión del marxismo recoge los intereses mediadores y orientadores del conocimiento, los contextualiza y los refiere a la totalidad de la existencia humana. Consecuentemente, para la teoría crítica, su ideal es la transformación del orden social y la búsqueda de un mayor grado de humanización. La ciencia debe servir así, al bienestar y a la transformación social de la humanidad, además de compartir el destino de las fuerzas y medios productivos como factor de riqueza de un país (Horkheimer, 1998).

El método debe estar, pues, en consonancia con el enfoque; es por eso que el procedimiento que presenta una mayor coherencia y validez con el enfoque crítico social, es el estudio de caso cualitativo que, como una totalidad holística refleja todos los elementos que componen dicha realidad, pero siempre considerada como una unidad. Un caso puede estar representado por una persona (un alumno, un profesor), una organización (un aula de clase o toda una institución educativa), un acontecimiento, una comunidad, un programa de enseñanza-aprendizaje, un plan de estudios 0 todo un currículo, que se investiga como una unidad (Marín, 2012).

En esta investigación, el tema se analizó como un programa de investigación, caracterizado por ser, en primer lugar, un estudio en profundidad de una unidad de observación (los programas de doctorado en educación en Colombia, 200-2012) y, en segundo lugar, porque el estudio de caso fue útil para obtener información básica que servirá, más adelante, para planear investigaciones mucho más amplias que puedan arrojar luz sobre importantes variables, interacciones y procesos merecedores de ser investigados más extensivamente (Tamayo y Tamayo, 1999). En síntesis, el estudio de caso cualitativo permite explorar, describir, evaluar, interpretar y, quizá, intervenir en algún momento la realidad.

La unidad de análisis estuvo constituida por las propuestas de las líneas de investigación y los trabajos que han realizado los grupos de investigación y los doctorados en educación en sus tesis, desde el 2000 a 2010. Se examinaron solamente tres de los programas de Doctorado en Educación que tienen productos de investigación: la Red RudeColombia (8 universidades del país), el interinstitucional 
(Pedagógica, Distrital y la Universidad del Valle) y la Universidad de Antioquia. De los nuevos doctorados que fueron creados a partir del 2009, como el Doctorado en Educación de la Universidad Santo Tomás, la Universidad de la Salle y la Universidad de los Andes, sólo se tuvieron en cuenta los documentos que se encontraron en la Red (páginas Web), para examinar sus propuestas investigativas, de acuerdo con sus líneas de investigación, por cuanto todavía no tienen productos que fueran resultado de sus investigaciones.

La técnica de recogida de la información pertinente y coherente con el método empleado fue la observación y el examen de los documentos, como una técnica que se usa en investigaciones diseñadas para obtener una descripción sistemática o para demostrar la hipótesis planteada. La observación facilitó y permitió determinar los aspectos relevantes para alcanzar los objetivos y propósitos investigativos.

\section{Resultados de la investigación}

La investigación inició propiamente con una contextualización y descripción de las condiciones de los doctorados en Colombia y, específicamente, en el área de las Ciencias de la Educación, ubicando en este contexto el problema de los doctorados y maestrías. Para ello se consultaron fuentes como el diagnóstico en Competitividad, desarrollo científico, tecnológico e innovación de Zulli David Hoyos, presentado en Santa Marta en septiembre de 2006 durante el Encuentro departamental de educación técnica y tecnológica, que muestra las tendencias de la formación doctoral para el siglo XXI. El proyecto Red de observatorios de buenas prácticas de dirección estratégica universitaria en América Latina y Europa (Informe Colombia, 2011), financiado con recursos Alfa de la Comunidad Económica Europea, que presenta estudios actuales cercanos a un panorama más real de la formación avanzada en el país.

Se consultó también el estudio Situación actual de los doctorados en Colombia: análisis de los indicadores que tipifican características importantes, del Portal del Ministerio de Educación Nacional, Colombia Aprende, en el que la reflexión que se hace, se centra en cómo las características de los doctorados deben contar con apoyo y fomento para su extensibilidad y calidad. Se proyectan, entonces, para el periodo (2008-2019), metas importantes que se esperan alcanzar con el enfoque del nuevo sistema de investigación y desarrollo en CT+ID .

6 Ciencia, Tecnología, Innovación más Desarrollo.

ISSN: 1657-107X, RIIEP, Vol. 7, N. ${ }^{0} 1$, enero-junio de 2014, pp. 105-103 
Estos informes revelaron datos importantes en la investigación: por una parte, el sistema de la Educación Superior se vuelve cada vez más complejo, pero por otra, la cobertura no aumenta, ni incrementa la inclusión y la posibilidad de acceder al sistema de los grupos poblacionales con dificultades sociales y económicas.

De esta primera parte de contextualización y descripción de las condiciones de los doctorados, se concluye con lo que se dice en el texto de Ciencia y tecnología en las universidades iberoamericanas, de Mario Albornoz y José Antonio López Cerezo (2010), en el que se plantea que hablar hoy de investigación científica y desarrollo ha perdido todo sentido, pues, la creencia de que a mayor educación mayor desarrollo y bienestar, se viene desdibujando en la realidad geopolítica mundial, con base en las últimas crisis del capital financiero transnacional que rompió con las lógicas analíticas y de crecimiento del mercado planetario, pues esta crisis se percibe actualmente, en los países de Europa y en los Estados Unidos, en donde se supone que los niveles de educación son unos de los más altos del planeta.

Una vez ubicados los doctorados dentro de su contexto situacional, la investigación continuó con el estado de la cuestión o estado del arte y con la descripción de los modelos pedagógicos subyacentes en las prácticas de formación investigativa en la formación de tercer nivel. Para ello, se tuvo en cuenta, en primer lugar y principalmente, los estudios sobre Estado de la investigación en pedagogía y educación (2009) de Myriam Henao, en los que se plantea que la educación ha construido su campo de acción como objeto complejo de estudio que convoca el interés y la preocupación de docentes, pensadores e investigadores, cuyos desarrollos constituyen el cuerpo de conocimientos que forman el estado de arte de la investigación educativa y pedagógica para el contexto colombiano. Asimismo, se tuvieron en cuenta los estudios de Olga Lucía Zuluaga y su grupo de investigación, sobre la Historias de las prácticas en educación, en los que se hallan los aportes de la historia como disciplina que articula los estudios genealógicos y arqueológicos de la educación en el desarrollo del país. En un segundo lugar y para los modelos pedagógicos, se acudió al concepto de paradigma de Thomas Kuhn (1992), en el sentido de que los modelos pedagógicos que subyacen a estas líneas de investigación en educación, son cercanos a las finalidades que tengan las líneas, los grupos y los énfasis investigativos de cada programa doctoral, en sintonía directa con el de las facultades e instituciones de educación superior en los que se encuentre hospedado. Pero de forma general, sea cual fuere este modelo de pensamiento, la educación en ciencias y las preguntas sobre la naturaleza de la ciencia y su manera de difundirlo y generarlo es inevitable. Es por esto que las universidades más fuertes 
en investigación parecen tener clara la necesidad de impulsar sus campos de saber con el ejercicio sistemático y continuado de la práctica investigativa, añadiendo un valor a la figura de los docentes-investigadores, no solo en el ejercicio de la investigación formativa, sino también en los procesos de investigación denominados científicos, o investigación propiamente dicha.

En síntesis, los modelos cognitivos, conceptuales, interactivos e investigativos, son los que actualmente articulan, de forma más coherente y viable, los proyectos de formación con los de investigación y los de proyección social al interior de los escenarios universitarios. Lamentablemente, la organización administrativa de la docencia y su carácter funcional, muchas veces no posibilita, de forma expedita, esta articulación, pues, se paga a los docentes-investigadores por horas de clase dictadas, y no por horas docencia/investigadas/extendidas/ problematizadas.

Vista la problemática de la formación doctoral en Colombia, el núcleo principal de la investigación consistió en el análisis de la información recolectada en los programas de doctorado en educación en Colombia y en la que se examinan, tanto las fuentes primarias como las fuentes secundarias en dos momentos metodológicos. En el primero se examinan los documentos maestros y otros textos de los doctorados en educación en Colombia que se encuentran en las páginas Web, con el fin de hallar en ellos la pertinencia de las propuestas de solución a los problemas de la educación, y qué tanto contribuyen al desarrollo y cambio social del país en forma sostenible.

En el segundo momento, se buscan las tendencias de las investigaciones realizadas por los proyectos de los grupos de aquellos doctorados que ya tienen investigaciones concluidas, como los de la red RudeColombia, el Interinstitucional y la Universidad de Antioquia, así como otros proyectos, tales como el presentado en Manizales por el Centro de Estudios Avanzados en Niñez y Juventud (CINDE) Universidad de Manizales, en su doctorado de Infancia y Juventud, denominado Regiones Investigativas en Educación y Pedagogía en Colombia (mayo, 2012), estudio dirigido por los investigadores principales, Héctor Fabio Ospina Serna y Napoleón Murcia Peña, en colaboración con otros investigadores nacionales.

Para una completa comprensión del fenómeno investigativo de los doctorados en educación y de su contribución al desarrollo de la ciencia, tecnología y sociedad (CTS), se hizo un recorrido por las diferentes tendencias investigativas a partir de las propuestas que se hallan en los documentos maestros de los programas de doctorado, de manera especial, leyendo los objetivos y el desarrollo de las líneas de investigación tal como se ofrecen. En términos generales, se encuentran frecuentes 
referencias comunes a la importancia de formar investigadores científicos, críticos, éticos, solidarios e innovadores que contribuyan al avance del conocimiento en el campo educativo y pedagógico y a formar redes de investigación. Los temas de investigación más comunes, se centran principalmente en las relaciones con la didáctica de las disciplinas, los Derechos Humanos, evaluación de la educación, gestión de la educación, historia de las prácticas pedagógicas y en el desarrollo social, entre otros. No se encuentran en estos documentos, muchas referencias a la proyección internacional, a la búsqueda de soluciones a los grandes conflictos y problemas de la globalización, a la protección y conservación del medio ambiente, al desarrollo tecnológico sostenible, a la integración educativa de los países latinoamericanos, a la violencia y la exclusión, como los problemas macros de la educación. Los objetos de investigación en los doctorados, se quedan en aspectos tradicionales y domésticos de la educación y la pedagogía.

En el segundo momento, una vez que se hubo analizado la información recolectada en las ventanas de observación específicas, alrededor de las líneas, los grupos y los proyectos de los doctorados en educación, en un primer acercamiento y con relación a la información proporcionada, las tendencias de las investigaciones y su continuidad con las líneas que se replican en los siguientes conceptos: Las didácticas, relacionadas con el enfoque de enseñanza de las ciencias y los saberes, acumuladas en el aspecto de formas de enseñar/aprender o de enseñar para aprender y que han avanzado en el tema de las mediaciones y racionalidades pedagógicas, conectadas con las racionalidades científicas.

La historia de los saberes y de las prácticas, consolidada por el grupo de Historia de las prácticas pedagógicas, (Grupo de Olga Lucía Zuluaga), con más de 32 años de existencia y ampliada con indagaciones de los saberes de filosofía, historia, antropología; así como otros aspectos relacionados con el aprendizaje e-learning y TIC y las ciencias experimentales y exactas.

La Sociedad-Educación, comprende aspectos de formación ciudadana, estudios políticos aplicados a la educación, estudios de cultura, capitales simbólicos y apropiaciones sociales, estudios ambientales aplicados educativamente; educación de adultos, educación popular e intercultural. Estos grupos empiezan a tener fuerza y acogida en los enfoques de ciencia, tecnología, naturaleza y sociedad (CTNS).

La Educación-Arte-Cultura, es uno de los campos de investigación, formación e intervención cercanos a la creación y a la sistematización de la expresión artística, con niveles de exploración comprehensiva e investigación formativa y propiamente dicha, en los programas de doctorado. 
El Aprendizaje e-learnig, software y TIC. Estas investigaciones, aunque de forma incipiente, pero creciente han dado lugar a temáticas en las líneas de investigación, comprendiéndolas no exclusivamente como herramientas, sino como formas mediáticas, con todas las implicaciones semióticas y cognitivas que le competen a las teorías educativas en sus aspectos de estilos de aprendizaje.

El Currículo, cuya teoría avanza en propuestas de organización interdisciplinar, de los contenidos seleccionados como enseñables y en una concepción amplia que lo vincula con otras disciplinas para problematizarlo con sentidos epistemológicos, sociales y económicos, pertinentes para la época.

La Cognición. Cercana al aprendizaje y a las influencias de la psicología; es un campo temático interdisciplinar en el que la pregunta sobre procesos de pensamiento se vincula, estrechamente, con procesos de enseñanza- aprendizaje.

La cognición compleja, mediática, simultánea, producto de la interacción simbólica, ha problematizado sus desarrollos teórico/metodológicos con tendencias emergentes en las dos últimas décadas, desde la perspectiva de la transformación comportamental y en sintonía con los descubrimientos de las inteligencias múltiples (H. Gardner), los estilos de aprendizaje y la inteligencia emocional (Goleman ).

Con respecto al documento Regiones investigativas en educación y pedagogía en Colombia (mayo, 2012), el texto ofrece un seguimiento a los acumulados que son leídos e interpretados por los doctorados en educación desde sus diferentes posicionamientos epistemológicos, y hacen un reconocimiento al saber pedagógico, apropiando las producciones legadas por el grupo de Historia de las prácticas pedagógicas.

Los subtemas colaterales que se recogen en el documento (Ospina, Murcia, 2012, p. 130), y que se contrastan con los reportados por los grupos de investigación/ líneas/proyectos de los visibilizados en las Web de los doctorados en educación en el país, reiteran los saberes y prácticas pedagógicas, didácticas, currículo, evaluación educativa, actores y escenarios educativos, modalidades educativas, problemas sociales, culturales y educativos contemporáneos.

Por lo tanto, podría afirmarse que los doctorados en educación investigan temas similares a los que se exploran en las maestrías en educación; lo que varía, es su nivel de profundidad y desarrollo teórico. En este sentido, cabe preguntarse: ¿en qué han variado estos temas de investigación pedagógico/educativo en los últimos 30 años de evolución del campo intelectual de la educación? ¿Investigamos sobre la tradición o sobre nuevas emergencias? ¿Qué rupturas investigativas en teoría de la educación hemos generado? Muchos interrogantes se multiplicarían con relación a esta 
información suministrada por el ejercicio de Regiones investigativas en educación y pedagogía en Colombia, que ameritan comprensiones lentas y digeridas. No se descartan, sin embargo, temas como: configuración subjetiva y socialización política, afectividad y erotismo, desarrollo cognitivo, desarrollo físico madurativo y neurodesarrollo, desarrollo moral, comunicación y lenguaje, socialización y cultura, lúdica y estética, ecología humana y educación, y habilidades para la vida.

A la luz de estos datos, el análisis de contexto y sus incidencias siguen permeando la investigación educativa en la relación educación-sociedad, con la misma o mayor presencia que tuvo en las décadas de 1980 al 2000, y con las manifestaciones de impacto que tuvo el Movimiento Pedagógico Nacional y su continuidad en las innovaciones educativas, experiencias alternativas pedagógicas y la Expedición Pedagógica Nacional.

\section{Discusión}

El problema de investigación a partir de la pregunta formulada como problema al inicio de esta investigación, se plasmó así: ¿Cuáles son las tendencias investigativas de los doctorados en educación en Colombia desde 2000 a 2010, en el campo de la ciencia, la tecnología y sociedad, y qué aportes han hecho mediante la construcción de sus teorías pedagógicas, a la calidad de la educación y al desarrollo social y económico del país?

Ahora es la oportunidad de confrontar estos dos momentos de la investigación: el problema y sus resultados. Con respecto a las tendencias investigativas de los doctorados en Colombia en la década pasada, no hay duda que se logró determinar cuáles fueron esas tendencias, las que quedaron expuestas ampliamente en el cuerpo de la investigación. Con respecto a la segunda parte del problema, es decir, si estas tendencias, en los aspectos de ciencia, tecnología y sociedad han contribuido efectivamente con la construcción de teorías pedagógicas a la calidad de la educación y al desarrollo social y económico del país, todavía no se percibe, en forma clara y precisa, hasta dónde se han logrado esos objetivos. Harán falta nuevas investigaciones, y habrá que esperar hasta que los nuevos doctorados en educación comiencen a hacer visibles sus productos investigativos. No obstante, es necesario tener en cuenta que los impactos no son siempre inmediatos, y que los productos investigativos de los doctorados en educación apenas han alcanzado los cinco años desde que se produjeron. 
Pero, con todo lo anterior, vale la pena considerar que se ha vuelto un lugar común la identificación del contexto de mundialización, globalizaciones y apertura del mercado como una variable de incidencia para que la educación superior se actualice, se modernice, y se transforme en términos de los encargos sociales que la banca multilateral le determina. Los doctorados en educación no escapan a esta predeterminación. Estas determinaciones obligan a cambiar los modelos de pensamiento y, por supuesto también, los modelos de cognición y producción de conocimiento. Por un lado, el paradigma de estandarización y universalización científica y tecnológica, se impone como referente de desarrollo y progreso ideal (pese a sus descalabros ambientales y sociales), y por otro lado, se sostiene la propuesta alternativa de mejoramiento de la calidad de vida de los habitantes del planeta, con un ritmo de crecimiento sostenido, sustentable y renovable.

La visión de educación más conocimiento igual desarrollo, sigue teniendo vigencia en las políticas educativas en el nivel superior, con relación a las potencialidades estratégicas que tiene la educación en la competitividad; pero no se puede ser tan ingenuo de pensar que este enfoque de desarrollo permitirá alcanzar el mismo nivel de los países del Norte, como bien dice Claudio Rama (2006): "El nacimiento de la sociedad del conocimiento transforma el saber en una mercancía, y su posesión, en un instrumento de poder geopolítico y de desarrollo económico" (p. 213).

Guarín Salazar y Mayorga Rincón (2008), en su texto Competitividad Internacional de la Educación Superior en la región de Bogotá y sus municipios circundantes, hacen un análisis de la capacidad de las IES en el sentido de sus ventajas comparativas desde la homologación con un par internacional, y sitúan la evaluación de las brechas de las capacidades institucionales, entre otras, bajo las siguientes condiciones:

- Baja cualificación de la mano de obra.

- Brecha tecnológica.

- Falta de integración de la educación superior con el sector empresarial.

- Débil capacidad de gestión.

- Baja inversión de capital en el sector.

- Vacíos en cooperación e investigación.

- Reducida infraestructura comunicacional necesaria para la construcción de comunidades académicas.

- Bajo número de reconocimientos internacionales y publicaciones en revistas indexadas.

- Ausencia de enfoques transdisciplinares vinculados a las necesidades de las mayorías poblacionales. (Salazar y Mayorga, 2008, pp. 44-45) 
El actual panorama exige, por lo tanto, una mirada realista que no se acerque en absoluto al derrotismo, sino a la exploración de otros horizontes de desarroIlo, como tanto se ha promovido en el enfoque de desarrollo a escala humana, o en el de desarrollo sostenible y sustentable. Producir ciencia sí, ¿pero de qué forma la enfocamos y la determinamos en un contexto ambiental de pervivencia y construcción de sociedad y ciudadanía, como indicador de civilidad y calidad de vida?

Esta pregunta es difícil de responder desde los alcances que esta investigación ha encontrado hasta el momento; pero en términos de la emergencia de proyectos de investigación en educación superior, modernización, actualización tecnológica y cognitiva, es obvio que el horizonte del discurso investigativo va encaminado hacia la confirmación de cumplir los encargos que mejoren los mercados laborales de la educación, con la cualificación en formación avanzada de todos los docentes y de todas las disciplinas en las universidades colombianas.

\section{Conclusiones}

Sin duda, en la forma como se dejó dicho en el proyecto de investigación, del que este artículo es apenas un resumen, los doctorados en educación necesitan abrir sus fronteras nacionales e internacionales en interés de lo disciplinar a los temas sociales y económicos en competencias ciudadanas, educación para la salud, escenarios virtuales de aprendizaje y currículos por competencias no solamente laborales, sino también para la vida; todo esto es lo que se valida de acuerdo con la responsabilidad social de la educación, aunada a la pertinencia exigida en el marco de la calidad exigida por la sociedad. Otros campos como la etnoeducación, la educación social, la educación intercultural, la educación para la diversidad, la educación con enfoque inclusivo, la educación ambiental, son también ámbitos de investigación que siguen avanzando en modos de gestión de procesos de enseñanza/aprendizaje, tanto en los escenarios institucionales educativos, como en los comunitarios que han dejado una impronta en la teoría educativa nacional, con la implementación de prácticas de democracia escolar, formación ciudadana, escuela nueva, escuela de adultos e integración educativa.

Las instituciones universitarias tienen, hasta cierto punto, la responsabilidad colectiva sobre la forma en que ayudan a construir las sociedades, por ser las 
que más contribuyen al avance y a la expansión masiva de la ciencia y la tecnología. Hace falta desarrollar estrategias desde los programas de doctorado en educación que vinculen la I+D con las demandas sociales. Estas estrategias deberán apuntar:

\begin{abstract}
"A que la selección de prioridades en I+D tome en cuenta aquellas que responden a necesidades concretas de la sociedad o a prioridades establecidas por las políticas sociales, así como a garantizar la transferencia y aplicación de los resultados. Esto implica la conformación de redes con actores diversos, la articulación con usuarios, la puesta en práctica de nuevos mecanismos de vinculación, el impulso a tecnologías sociales y el estímulo a la innovación social". (OEI, 2012, p. 71)
\end{abstract}

Para la Unesco, la educación superior cumple funciones sin precedentes en la sociedad actual, como componente esencial del desarrollo cultural, social, económico y político, y como elemento clave del fortalecimiento de las capacidades endógenas, la consolidación de los derechos humanos, el desarrollo sostenible de la democracia y la paz, en un marco de justicia. La educación superior ha de velar para que prevalezcan los valores ideales de la cultura de paz (Haddad, 2008). Sin embargo, aunque las universidades disponen de un potencial enorme para fomentar el desarrollo tecnológico, en la forma como lo comentan Calestous Juma y Lee-Yee Cheong en Innovación: aplicación de los conocimientos al desarrollo (2005),

"La mayor parte de estas instituciones de los países en desarrollo no cuentan con los equipos suficientes para encarar el reto, porque todavía poseen planes de estudio obsoletos, profesorado poco motivado, gestión inadecuada y una lucha continua por los fondos; todo esto ha llevado a minar la capacidad de las universidades para cumplir su función como motores de la comunidad o del desarrollo regional". (124)

Por último, no solamente la responsabilidad es de las instituciones de educación superior en abstracto, sino también de cada uno de los doctorandos en educación como parte esencial de estas instituciones; por lo tanto, caben las siguientes preguntas: ¿qué actitud asumen con sus investigaciones? ¿Qué aportes hacen al desarrollo de la comunidad y del país al cual pertenecen? 


\section{Referencias Bibliográficas}

Aibar, E. y Quintanilla, M. A. (editores). (2012). Ciencia, tecnología y sociedad. Madrid: Trotta.

Albornoz, M y López Cerezo J. A. (editores). (2010). Ciencia y Tecnología en la Universidades Iberoamericanas. Buenos Aires: Eudeba.

Guarín Salazar, K., y Mayorga Rincón, L. (2008). Competitividad Internacional de la Educación Superior en la región Bogotá y sus municipios circundantes. Bogotá: Universidad Cooperativa de Colombia.

Global University Network for Innovation. (2008). Educación Superior en el Mundo 3 Educación Superior: Nuevos Retos y Roles Emergentes para el Desarrollo Humano y Social. Madrid: Mundi-Prensa.

Haddad, G. (2008). La educación superior, agente del desarrollo humano y social: el punto de vista de la Unesco. En Global University Network for Innovation. Educación Superior en el Mundo 3. Educación Superior: Nuevos Retos y Roles Emergentes para el Desarrollo Humano y Social, p. xxxvii. Madrid: Mundi-Prensa.

Henao Willes, M. (2009). Estado de la investigación en Pedagogía y educación. Bogotá: Colciencias.

Hoyos, Z. D. (2006). Competitividad, Desarrollo Científico, Tecnológico e Innovación. Santa Marta: Encuentro departamental de educación técnica y tecnológica.

Horkheimer, M. (1998). Teoría crítica. Buenos Aires: Amorrortu editores.

Juma, C. y Yee-Cheong, L. (2005). Innovación: aplicación de los conocimientos al desarrollo. Bogotá: Colciencias.

Kuhn, T.S. (1992). La estructura de las revoluciones científicas (1ª reimp.) Bogotá: Fondo de Cultura Económica.

Marín Gallego, J. D. (2012). La investigación en educación y pedagogía. Sus fundamentos epistemológicos y metodológicos. Bogotá (en prensa).

Morell Alonso, D. (2009). Formación del profesorado de Ciencias Agronómicas de la Universidad Cubana de Ciego de Ávila en educación-Ciencia- Tecnología y Sociedad. Tesis Doctoral

OEI. (2008). Metas educativas 2021. La educación que queremos para la generación de los bicentenarios. Madrid: Estados Iberoamericanos para la Educación, la Ciencia y la Cultura.

OEI. (2012). Ciencia, tecnología e innovación para el desarrollo y la cohesión social: Programa iberoamericano en la década de los bicentenarios. Madrid: Estados Iberoamericanos para la Educación, la Ciencia y la Cultura. 
Ospina Serna, H., y Murcia Peña N. (2012). Regiones investigativas en educación y pedagogía en Colombia. Manizales: Zapata.

Rama, C. (2006). La tercera reforma de la educación superior en América Latina. México: Fondo de cultura Económica.

Tamayo y Tamayo, M. (1999). La investigación. Serie aprender a investigar (3a ed.). Bogotá: Icfes. 
